\title{
A modified technique of laryngotracheal reconstruction without the need for prolonged postoperative stenting
}

\author{
Konrad Hoetzenecker, MD, PhD, ${ }^{\mathrm{a}}$ Thomas Schweiger, MD, ${ }^{\mathrm{a}}$ Imme Roesner, MD, ${ }^{\mathrm{b}}$ \\ Matthias Leonhard, MD, ${ }^{\mathrm{b}}$ Gabriel Marta, MD, ${ }^{\mathrm{a}}$ Doris M. Denk-Linnert, MD, ${ }^{\mathrm{b}}$ \\ Berit Schneider-Stickler, MD, ${ }^{b}$ Wolfgang Bigenzahn, $\mathrm{MD}^{\mathrm{b}},{ }^{\mathrm{a}}$ and Walter Klepetko, $\mathrm{MD}^{\mathrm{a}}$
}

\begin{abstract}
Objectives: Repair of laryngotracheal stenosis with pronounced side-to-side narrowing and involvement of the glottis is challenging and usually requires laryngotracheal reconstruction with rib cartilage interpositions. This technique, as first described by Couraud, needs prolonged postoperative stabilization with Montgomery T-tubes, imposing significant morbidity and discomfort on patients. We describe our initial experience with a modified laryngotracheal reconstruction technique that avoids the need for prolonged postoperative stenting.
\end{abstract}

Methods: From November 2012 through May 2015, a series of 5 adult patients with glottosubglottic stenosis were operated in our institution. All patients had pronounced scar formation in combination with advanced side-to-side narrowing extending up to the level of the vocal folds. Operative technique consisted of a complete anterior and posterior laryngeal split followed by rib cartilage interposition in the cricoid plate posteriorly to enlarge the glottosubglottic diameter. The lateral edges of the rib graft were trimmed in such a way that lateral flanges were created, which allowed stable positioning of the graft. The distal trachea was then slid into the larynx, and the posterior defect was completely covered with a liberal membranous flap. The anterior part of the larynx was enlarged with a V-shaped segment of the anterior tracheal wall.

Results: This technique provided immediate stability without the need for temporary endoluminal stenting. The perioperative course was uneventful in all patients, and functional outcome was excellent.

Conclusions: We conclude that this modified technique of laryngotracheal reconstruction represents a valid treatment option for patients with complex glottosubglottic stenosis, avoiding the need for prolonged postoperative stenting. (J Thorac Cardiovasc Surg 2016;152:1008-17)

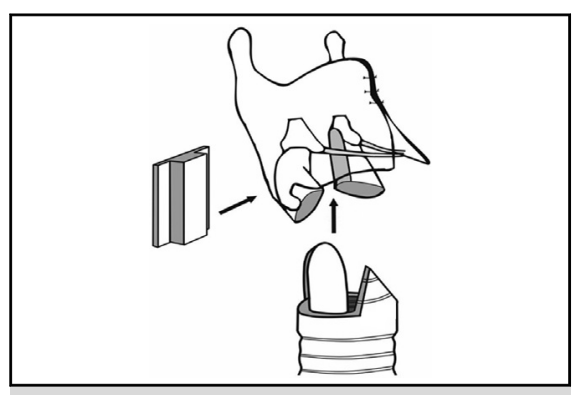

Scheme of the modified technique of laryngotracheal reconstruction.

\section{Central Message}

Herein presented modifications of the Couraud technique make laryngotracheal reconstructions feasible without prolonged postoperative T-tube stenting.

\section{Perspective}

Laryngotracheal reconstruction in adults usually requires a prolonged postoperative T-tube stenting for stabilization of the newly reconstructed airway. We present our first experience with a modified technique of laryngotracheal reconstruction that leads to an immediate stability and thus facilitates the repair as a single-stage procedure.

See Editorial Commentary page 1018.
Laryngotracheal stenoses in adults are an infrequent problem and require a sophisticated surgical approach to offer optimal results to affected patients. Stenoses restricted to the subglottic region can be repaired with excellent results by means of the Grillo or Pearson technique. ${ }^{1,2}$ When the subglottic stenosis extends to the level of the vocal folds,

From the ${ }^{\mathrm{a} D e p a r t m e n t}$ of Thoracic Surgery and ${ }^{\mathrm{b}}$ Division of Phoniatrics-Logopedics, Department of Otorhinolaryngology, Medical University, Vienna, Austria.

Read at the 96th Annual Meeting of The American Association for Thoracic Surgery, Baltimore, Maryland, May 14-18, 2016.

Received for publication Nov 8, 2015; revisions received Jan 1, 2016; accepted for publication Jan 17, 2016.

Address for reprints: Konrad Hoetzenecker, MD, PhD, Medical University of Vienna, Department of Thoracic Surgery, Währinger Gürtel 18-20, 1090 Vienna, Austria (E-mail: konrad.hoetzenecker@meduniwien.ac.at).

0022-5223/\$36.00

Copyright $($ C 2016 by The American Association for Thoracic Surgery

http://dx.doi.org/10.1016/j.jtcvs.2016.01.061 however, surgical repair becomes more challenging. ${ }^{3}$ In such a situation, classic subglottic resections do not provide satisfactory results, ${ }^{4}$ because these complex stenoses require not only a resection of all scar formations but also an enlargement of the airway lumen. Such a technique was originally described by Couraud and colleagues. ${ }^{5,6}$ They reported on 12 patients with severe glottosubglottic stenosis treated with a laryngeal enlargement. An anterior and a posterior vertical cricoidotomy was performed, and a 3- to 5-mm wide periosteal graft was inserted anteriorly

Scanning this QR code will take you to procedural videos.

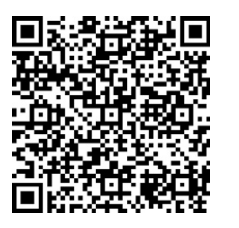




$$
\begin{aligned}
& \text { Abbreviations and Acronyms } \\
& \qquad \begin{aligned}
\mathrm{POD}= & \text { postoperative day } \\
\mathrm{R} & =[\text { vocal }] \text { roughness } \\
\mathrm{B} & =[\text { vocal }] \text { breathiness } \\
\mathrm{H}= & {[\text { vocal }] \text { hoarseness } } \\
\mathrm{MEF}= & \text { maximum expiratory flow } \\
\mathrm{ENT}= & \text { ear, nose, and throat } \\
\mathrm{FEES}= & \text { fiberoptic endoscopic evaluation of } \\
& \text { swallowing }
\end{aligned}
\end{aligned}
$$

and posteriorly between the two edges of the cricoid. The width of the reconstructed larynx was maintained by a Ttube, which remained in place for 3 months until complete healing and coverage by mucosal ingrowth had occurred. One of their patients died; the remaining 11 patients were reported to have good functional results with fair to good quality of voice. ${ }^{6}$ Additional information about this technique derives from a pooled Spanish experience, which reported on 20 patients treated the same way between 1986 and 2011. Results were described as good, and T-tubes could be removed after a mean of 60 days (range, 28-180 days). ${ }^{4}$ Thus the Couraud technique seems to offer satisfactory outcome, however, it carries the disadvantage of requiring prolonged postoperative stenting with Montgomery T-tubes until complete stabilization and mucosal coverage have been achieved.

In pediatric patients, laryngotracheal reconstructions represent an established and frequently applied technique, with series of more than 100 patients reported from Lausanne and Cincinnati. ${ }^{7,8}$ In this population, the cartilage grafts are trimmed with lateral flanges and sutured with 60 sutures to the two halves of the split cricoid plate. ${ }^{9}$ This maneuver leads to an immediately stable reconstruction of the naturally floppy pediatric airway. Nevertheless, the lack of mucosal coverage, as well as the difficult perioperative handling of small children, often makes the temporary implantation of a laryngotracheal mold for internal stenting necessary.

We therefore hypothesized that by adapting the pediatric technique of immediate stabilization and combining it with a complete coverage with healthy mucosa, laryngotracheal reconstruction in adults could be achieved in a single-stage procedure without the need for prolonged postoperative $\mathrm{T}$ tube stenting. This case series summarizes our initial experience with the first 5 patients who were treated in this way.

\section{MATERIALS AND METHODS \\ Patients}

From November 2012 to May 2015, a total of 75 laryngotracheal operations were performed in pediatric and adult patients in our department. Among these, 5 adult patients were found to have the unique disease entity of a glottosubglottic stenosis extending up to the level of the vocal folds. The type of the stenosis was mainly a side-to-side narrowing, so classic resection techniques (Pearson technique, Grillo technique) were not applicable. In all 5 patients, the airway, respiration, swallowing and phonation were preoperatively evaluated by transnasal flexible laryngoscopy. All patients (except patient 5, who had a permanent tracheostomy before the operation) underwent spirometry for functional quantification of the degree of upper airway obstruction (JAEGER Master Screen Body spirometer; PanGas AG, Dagmersellen, Switzerland). Flexible bronchoscopy under general anesthesia was performed to define precisely the type and the extension of the airway problem. In addition, imaging with a cervical computed tomographic scan was done in all cases. Preoperative, perioperative, and postoperative patient management was performed by a dedicated laryngotracheal team consisting of thoracic surgeons, phoniatricians, speech therapists, and anesthetists.

\section{Surgical Method}

Patients were positioned in the supine position with an overextended neck, and ventilation through a laryngeal mask was installed. Patient 5 was ventilated through the preexisting tracheostomy. Intravenous anesthesia was used for induction and maintenance of anesthesia. After cervical incision and mobilization of the strap muscles, the glottis, the subglottis, and the cervical trachea were dissected anteriorly and laterally as far as needed without impairing the blood supply too extensively. In patient 5 , the existing tracheostomy was incorporated into the skin incision. Lateral preparation was performed close to the trachea by blunt dissection or use of electrocautery at a very low energy level. Recurrent nerves were not routinely dissected, and recurrent nerve monitoring devices were not used. The trachea was then transected under bronchoscopic guidance at the lower end of the stenosis, and crosstable ventilation through the distal tracheal stump was initiated.

Resection of the diseased segment was started by removal of the cricoid arch. A complete anterior split of the thyroid cartilage was performed precisely in the midline, with the thyroid notch used as the leading structure to avoid lateral deviation and damage to the vocal folds. In the next step, all scar formations at the anterior surface of the cricoid plate were removed, either with the scalpel or with the help of an electric drill. Thereafter, the cricoid plate was completely split vertically in the midline, with the mucosal incision reaching up almost to the interarytenoid fold. The interarytenoid muscle was transected in case of scarring. The posteromedial parts of the two halves of the cricoid plate were carefully dissected posteriorly away from the hypopharynx to create a space for the later insertion of the cartilage graft. A rib cartilage graft was harvested through a 5-cm skin incision above the costal arch. Muscles were dissected and the cartilaginous part of a rib was isolated. Care was taken to avoid opening of the pleural space. A $3-\mathrm{cm}$ large rib was harvested, and the resulting defect was covered by muscles. The cartilage was trimmed to a width of 4 to $5 \mathrm{~mm}$, with 2 posterior flanges of 2 to $3 \mathrm{~mm}$ extending to both sides. This graft was snapped between the two cricoid halves, with the flanges extending on both sides posterior to the cricoid plate. The cartilage graft was secured with 4 to 6 stitches of 6-0 polydioxanone suture.

The distal trachea was then tailored in such a way that a long dorsal mucosal flap and an anterior V-shaped cartilage segment was created. A jet ventilation catheter was introduced through the laryngeal mask, crosstable ventilation was stopped, and the anastomosis was performed under jet ventilation (TwinStream; Carl Reiner GmbH, Vienna, Austria). In a first step, a running stitch of 5-0 polydioxanone suture was placed between the dorsal mucosal flap and the proximal dissection line of the glottic mucosa, without yet bringing the two parts into full contact. Stitches at the anterior part of the anastomosis were then prepared, and the two halves of the anterior thyroid cartilages were spread apart to allow interposition of the V-shaped distal anterior tracheal segment (Figure 1 and Video 1).

The distal trachea was then stepwise brought into direct contact with the laryngeal skeleton by gently pulling on the anterior single stitches. The posteriorly running suture was straightened until full adaptation and complete 



FIGURE 1. Photographs depict important surgical steps of the modified laryngotracheal reconstruction. A, After a complete anterior laryngeal split and resection of the cricoid arch, the side-to-side nature of the glottosubglottic stenosis can be seen. B, The cricoid plate is transected in the midline to facilitate an enlargement of the glottic diameter. C, Rib cartilage is harvested and trimmed with 2 flanges on both sides. D, The cartilage graft is inserted between the split parts of the cricoid plate and forms a stable reconstruction. E, The graft is covered by a dorsal mucosal flap with a running 5-0 polydioxanone suture. F, The anterior laryngeal split is closed, and a thyrotracheal anastomosis with the V-shaped distal trachea is performed.

coverage of the posterior cartilage graft, as well as the mucosal defect on the cricoid plate, had been achieved. Once the posterior adaptation was completed, the anterior stitches were knotted creating an airtight anastomosis.

At the end of the procedure, flexible bronchoscopy was performed to judge the quality of the anastomosis. In case of relevant swelling and edema of the vocal cords, a mini tracheostomy (size 5 or 6 ) was placed distal the anastomosis (patients 2, 3, and 5) for a few days. Patients were evaluated by transnasal flexible laryngoscopy on postoperative day (POD) 1 . When vocal fold movements were satisfactory and no aspiration occurred, oral nutrition was started. Patients, who had received a minitracheostomy at the end of the procedure, were decannulated once the swelling of the vocal folds had subsided and a sufficient glottis diameter was confirmed by transnasal flexible laryngoscopy.

\section{Voice and Swallowing Evaluation}

Subjective perceptive evaluation of voice quality was determined according to the parameters of roughness (R), breathiness (B), and hoarseness 


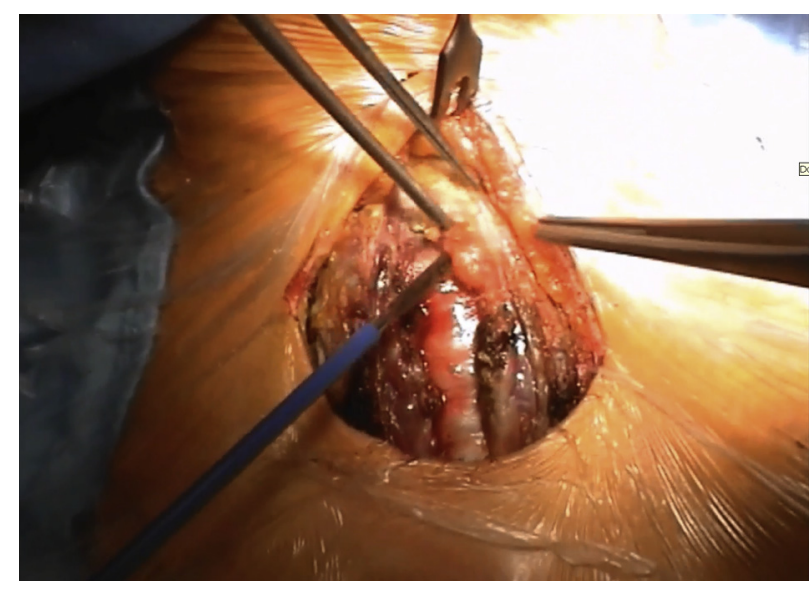

VIDEO 1. This video illustrates surgical steps of the modified laryngotracheal reconstruction technique. Video available at http://www.jtcvsonline. org/article/S0022-5223(16)30886-8/addons.

(H) and a grading of 0 (normal), 1 (slightly impaired), to 2 (moderate or severely impaired). Voice range profile was determined by means of the DIVAS voice diagnostics tool (mean fundamental frequency of speaking voice, dynamic range of singing voice, range of singing voice in semitones; XION Medical GmbH, Berlin, Germany) before and three months after the operation. Swallowing was documented by fiberoptic endoscopic evaluation of swallowing (FEES) before the operation and postoperatively (before starting enteral nutrition and 3 months after the operation). Findings of FEES were documented by means of a structured form developed in Vienna (D.M.D.-L., W.B.). For each consistency (liquid, semiliquid, solid food), the amounts of aspiration, penetration, and retention were evaluated.

\section{Follow-up}

After discharge from hospital, patients were followed up on an individual basis. In addition to that, every patient was seen by the laryngotracheal team 3 months after the operation, when a control bronchoscopy, a swallowing test, and a voice assessment were performed. Bronchoscopy was performed in general anesthesia to have optimal conditions for the examination, since coughing and natural movements of the larynx make a proper judgement of the glottic level difficult. Depending on the findings, further follow-up evaluations were scheduled.

\section{Statistical Methods}

The statistical software package IBM SPSS 21 (IBM Corporation, Armonk, NY) was used for the statistical analyses. All data are given as mean \pm SD. Preoperative and postoperative lung function parameters were compared with a 2 -sided dependent $t$ test for paired samples.

\section{RESULTS}

Between November 2012 and May 2015, a total of 5 adults were operated using the modified laryngotracheal reconstruction technique described above. Median age at the time of operation was 39 years (range, 26-45 years). Three patients suffered from an idiopathic stenosis. Two patients had a history of former intubation. Two patients had previous treatments before referral. Patient 4 had undergone a laryngoplasty with an anterior cartilage graft 5 years previously; however, this procedure had failed. Patient 5 had undergone 2 endoluminal laser ablations and dilatations 6 years and 4.5 years previously. A third attempt of laser enlargement failed, and a tracheostomy was placed before the patient was referred to our institution (Table 1). Severity and extent of stenoses were graded as IIIc according to the modified Myer-Cotton scale. ${ }^{10}$ All 5 patients had normal preoperative swallowing functions and vocal fold movements. The surgical procedure was performed as described in detail in the Materials and Methods section. The enlargement plasty with the dorsal cartilage graft was intraoperatively considered completely stable in all patients. Prolonged endoluminal splinting of the reconstructed airway with a stent, mold, or endotracheal tube was therefore not considered to be necessary. Three patients developed swelling and edema of the vocal folds at the end of the operation, and a minitracheostomy was therefore created distal to the anastomosis. All 3 of these patients were decannulated when the swelling had resolved, between POD 8 and POD 15. Oral nutrition was started after FEES on a median of POD 6 (range, POD 1-10). In none of the patients aspiration of fluid, semiliquid, or solid consistency was found postoperatively. Vocal cord movement was closely monitored after the operation, because both recurrent nerves are naturally placed at risk by the surgical procedure. One patient (patient 5) had an impaired movement of her vocal folds with fixation in a position of maximal abduction and therefore never experienced breathing problems. She had no impaired swallowing, and after logopedic training she developed a nearly normal voice quality with her false vocal folds. In patient 3 , a bilateral incomplete abduction was diagnosed, which did not lead to impaired breathing. Respiratory vocal fold movement was completely normal in patients 1,2 , and 4 (Table 2).

TABLE 1. Preoperative data

\begin{tabular}{llcllcc}
\hline Patient & Sex & Age $(\mathbf{y})$ & \multicolumn{1}{c}{ Etiology } & Pretreatment & $\begin{array}{c}\text { Stoma at } \\
\text { operation }\end{array}$ & $\begin{array}{c}\text { Modified } \\
\text { Myer-Cotton grade }\end{array}$ \\
\hline 1 & F & 26 & Idiopathic & None & No & IIIc \\
2 & M & 34 & Postintubation stoma & None & Yes & IIIc \\
3 & M & 41 & Idiopathic & None & No & IIIc \\
4 & F & 39 & Postintubation stoma & Tracheoplasty & IIIc \\
5 & F & 45 & Idiopathic & Laser $(\times 2)$ & Yes & IIIc \\
\hline
\end{tabular}


TABLE 2. Surgical procedure

\begin{tabular}{|c|c|c|c|c|c|c|c|c|c|}
\hline Patient & Approach & $\begin{array}{c}\text { Cartilage } \\
\text { enlargement }\end{array}$ & $\begin{array}{r}\text { Operation } \\
\text { time (min) } \\
\end{array}$ & $\begin{array}{l}\text { Swelling of } \\
\text { vocal cords }\end{array}$ & $\begin{array}{c}\text { Prolonged } \\
\text { stoma } \\
\end{array}$ & $\begin{array}{c}\text { Start oral } \\
\text { feeding }\end{array}$ & Discharge & Decannulation & $\begin{array}{l}\text { Vocal cord } \\
\text { movement }\end{array}$ \\
\hline 1 & Cervical & Posterior & 255 & No & No & POD 2 & POD 5 & NA & Normal \\
\hline 2 & Cervical & Posterior & 285 & Yes & Yes & POD 7 & POD 12 & POD 8 & Normal \\
\hline 3 & Cervical & Posterior & 225 & Yes & Yes & POD 1 & POD 4 & POD 14 & $\begin{array}{l}\text { Bilateral incomplete } \\
\text { abduction }\end{array}$ \\
\hline 4 & Cervical & Posterior & 275 & No & No & POD 5 & POD 8 & NA & Normal \\
\hline 5 & Cervical & Posterior & 285 & Yes & Yes & POD 10 & POD 15 & POD 9 & $\begin{array}{l}\text { Impaired bilateral } \\
\text { movement }\end{array}$ \\
\hline
\end{tabular}

POD, Postoperative day; $N A$, not applicable.

\section{Anastomotic Healing}

Anastomoses were monitored by flexible bronchoscopy before discharge from hospital and 3 months after the operation. Healing was excellent in all cases. No evidence for granulation formation was found, and only an inert, small scar line was visible 3 months after the reconstruction (Figure 2 and Video 2). In patients 2 and 5, remnants of the incompletely dissolved polydioxanone suture were removed with a flexible forceps. In patient 4 , the anterior split of the thyroid healed in a displaced way with left vocal cord inserting $1 \mathrm{~mm}$ higher than the right. The patient phonated with her left true vocal fold and her right ventricular fold.

\section{Lung Function}

Lung function parameters were significantly impaired preoperatively according to the severity of the airway obstruction. Peak flow was $41.2 \pm 13.1$ (\% predicted; mean $\pm \mathrm{SD}$ ), maximum expiratory flow (MEF) after $75 \%$ exhalation of the vital capacity (MEF 75) was $51.0 \pm 15.3 \%$ predicted and MEF after $50 \%$ exhalation of the vital capacity (MEF 50) was $60.4 \pm 14.7 \%$ predicted. Postoperative lung function parameters were significantly improved, with a peak flow of $98.7 \pm 20.6(P=.033)$, MEF 75 of $103.5 \pm 21.5(P=.007)$, and a MEF 50 of $82.0 \pm 20.3(P=.022$; Figure 2$)$.

\section{Voice Quality}

Voice quality was monitored before and after the operation in 4 of our 5 patients. All patients received logopedic training after hospital discharge. Voice evaluation serving as the final functional result was performed 3 months after the reconstruction. Subjective voice quality was found to be excellent or satisfactory by all patients. In 2 patients (patients 1 and 2), voice range and dynamic range, as well as fundamental frequency of the speaking voice, were unaffected by the reconstruction. Patient 1 had a mild increase in roughness, breathiness, and hoarseness (preoperative assessment, R0 B0 H0; postoperative assessment, R1 B1 H1). Perceptual voice evaluation was unchanged in patient 2 (preoperative assessment, $\mathrm{R} 0 \mathrm{~B} 0 \mathrm{H} 0$; postoperative assessment, R0 B0 H0). Patient 4 had a significantly impaired postoperative voice quality, corresponding to the displaced insertion of the vocal folds after the procedure (preoperative assessment, R1 B0 H1; postoperative assessment, R3 B2 H3, diplophonic). She phonated with her left true vocal fold and the right false vocal fold. As a consequence, her fundamental voice frequency dropped by 4 semitones, and her voice range and dynamic range deteriorated. Patient 5 developed a supraglottic phonation that used her ventricular folds. This resulted in a reduction of her fundamental frequency; however, dynamic range and voice range were unchanged relative to preoperative values (Table 3 ).

\section{DISCUSSION}

The principles of tracheal surgery were established in the $1950 \mathrm{~s}^{11}$; however, resection techniques involving the subglottic region developed much later. The Pearson and Grillo techniques were first published in 1975 and 1982, respectively. ${ }^{1,2}$ Both techniques led to excellent long-term results in patients with an anterior or circular type of stenosis. A high rate of failure was seen for stenosis with a mainly side-to-side narrowing and an extension of scar tissue up to the level of the vocal cords. For this type of problem, Couraud and colleagues ${ }^{5,6}$ suggested a technique that combines a subglottic resection with an enlargement of the glottis, which is achieved by a complete anterior and posterior split of the larynx and interposition of an anterior and posterior periosteal (hyoid) graft. This procedure, however, implies a certain degree of destabilization of the laryngeal skeleton and, even more, leaves the surface of the grafts devoid of mucosal coverage. For both reasons, a prolonged postoperative stabilization with a Montgomery T-tube ${ }^{6}$ is necessary until stability has been achieved and coverage of the denuded cartilage has occurred through mucosal ingrowth. ${ }^{12}$ The usually recommended period of such an internal stenting is 3 to 6 months.

From this background, it becomes clear that any modification of this technique that could avoid the need for postoperative stenting must address both problems, stability and mucosal coverage. From the pediatric population, it is well known that the use of costal cartilage grafts with lateral steps provide a firm contact with the cricoid plate and thus results in a stable reconstruction. On the other hand, 


\section{pre-operatively}
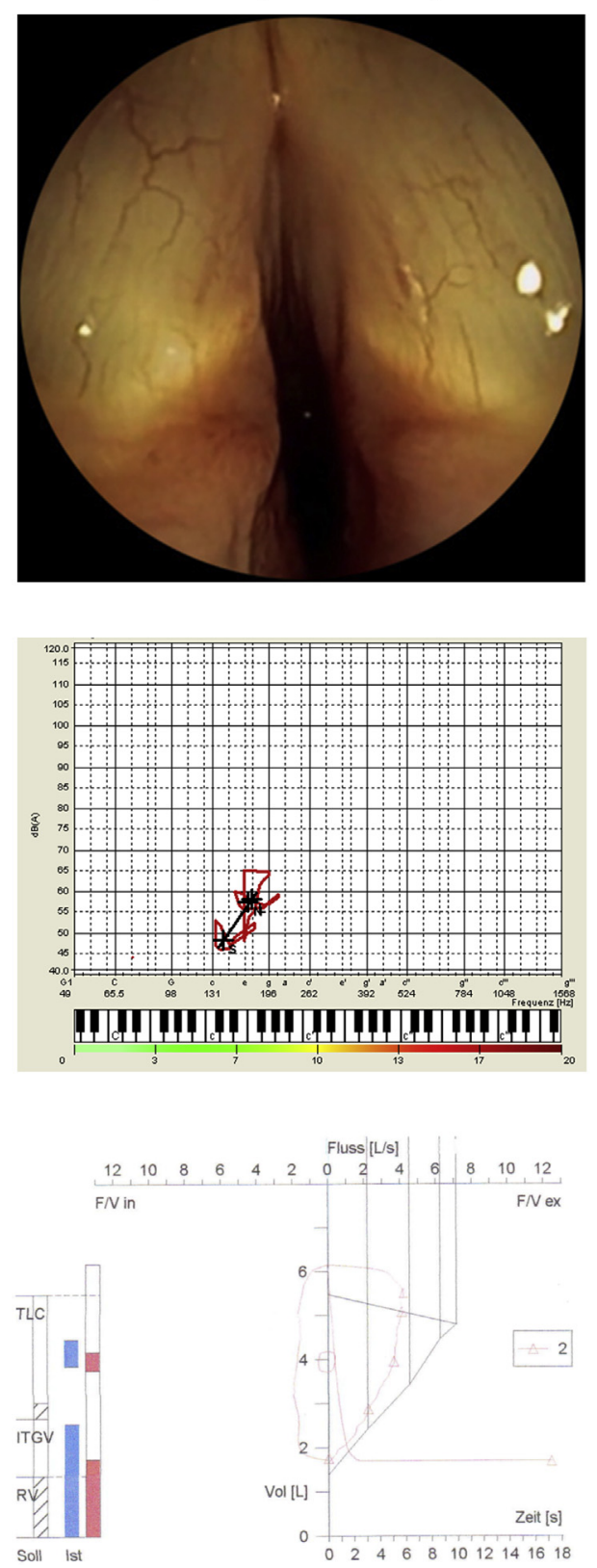
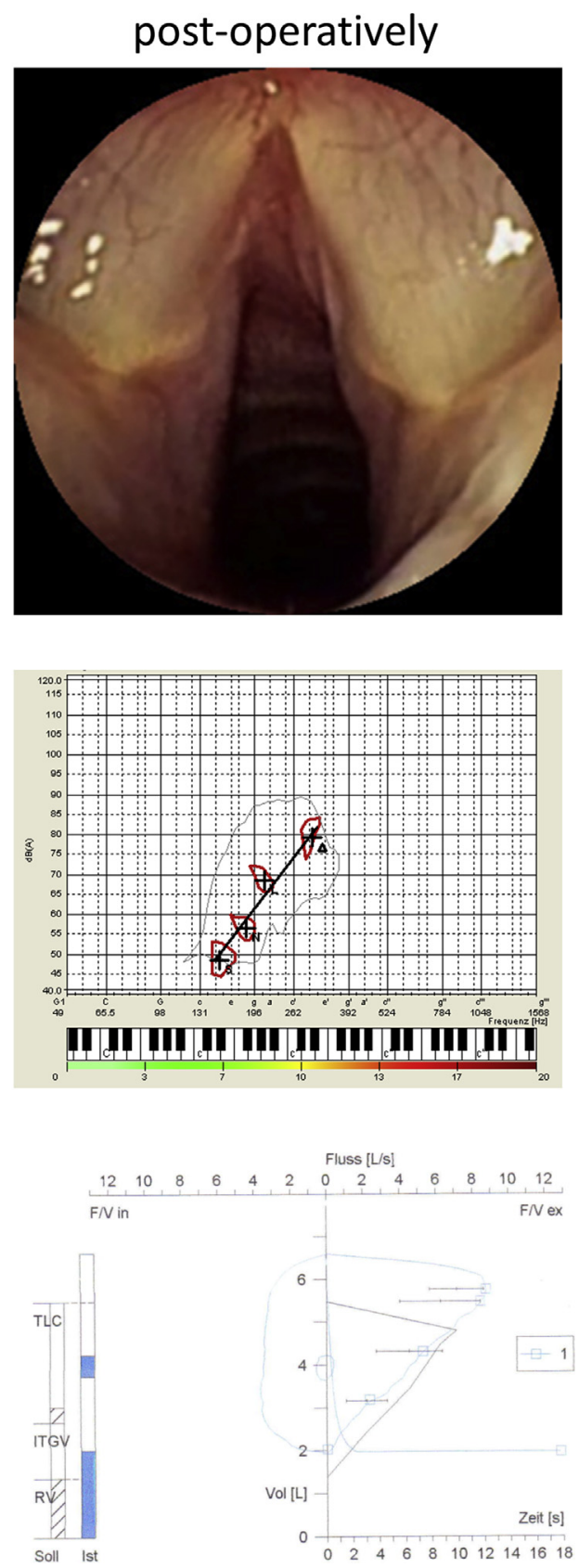

FIGURE 2. Preoperative (left) and postoperative (right) bronchoscopic findings (top), voice range tests (middle), and lung function tests (bottom) of patient 1. A significant enlarged airway diameter led to a profound improvement of lung function parameters. $F / V$, Flow/volume; $T L C$, total lung capacity; $I T G V$, intrathoracic gas volume; $R V$, residual volume.

the use of pedicled flaps from the posterior membranous portion of the trachea for coverage of the cricoid plate is an established technique used in cricotracheal resections. We therefore speculated that the combination of these techniques might result in an immediate stabilization and good mucosal healing. In addition, we used the anterior part of the distal trachea for the anterior enlargement of the larynx by interpositioning a $\mathrm{V}$-shaped segment instead of using a free costal cartilage graft. The use of the native trachea for anterior interposition has the clear advantage of being already covered with healthy mucosa and leading to a more stable anterior reconstruction (Figure 3).

Some other technical details are of importance for a successful repair. The thickness of the cartilage graft must be equal to that of the cricoid plate, and the width of the graft must be carefully chosen. Usually it should be between 3 and $5 \mathrm{~mm}$ maximum, because its dimension has a clear impact on postoperative voice quality. In fact, all our 


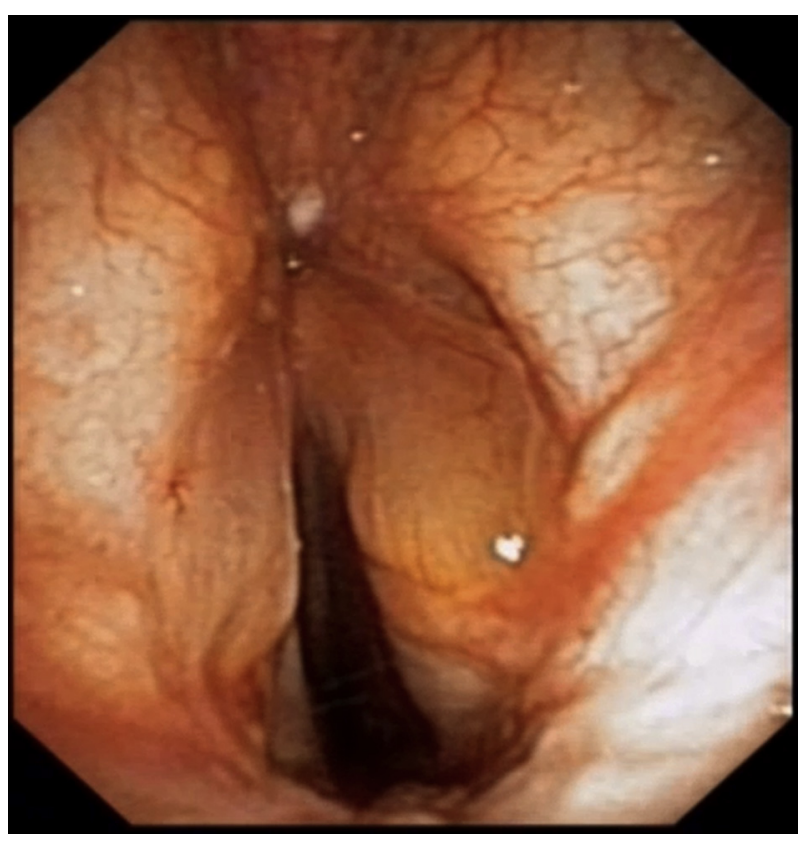

VIDEO 2. Video shows preoperative extent of the stenosis and postoperative result after reconstruction. Video available at http://www.jtcvsonline. org/article/S0022-5223(16)30886-8/addons.

patients had a decrease in their fundamental frequency; however, this decrease was not of clinical importance.

Furthermore, the careful adaptation of the posterior mucosa flap is of crucial importance for achieving good mucosal coverage and undisturbed healing. Prepositioning of the running suture line, followed by careful adaptation once the anterior part of the reconstruction is brought closer together, allows such a precise adaptation.

The fate of autologous cartilage graft transplantations has been highlighted in only few studies. There is a general understanding that the graft undergoes dramatic changes with

TABLE 3. Voice evaluation

\begin{tabular}{|c|c|c|c|c|}
\hline Patient & $\begin{array}{l}\text { Perceptual } \\
\quad \text { voice } \\
\text { evaluation }\end{array}$ & $\begin{array}{c}\text { Voice range of } \\
\text { singing voice } \\
\text { (semitones) }\end{array}$ & $\begin{array}{l}\text { Dynamic } \\
\text { range } \\
\text { of singing } \\
\text { voice }(d B)\end{array}$ & $\begin{array}{c}\text { Fundamental } \\
\text { frequency of } \\
\text { speaking } \\
\text { voice }(\mathrm{Hz})\end{array}$ \\
\hline \multicolumn{5}{|c|}{ Preoperative } \\
\hline 1 & R0 B0 Ho & 26.0 & 50.0 & 197.8 \\
\hline 2 & R1 B0 H1 & 13.0 & 44.0 & 74.8 \\
\hline 4 & R1 B0 H1 & 41.0 & 30.0 & 209.6 \\
\hline 5 & R1 B0 H1 & 13.0 & 23.0 & 242.0 \\
\hline \multicolumn{5}{|c|}{ Postoperative } \\
\hline 1 & R1 B1 H1 & 20.0 & 45.0 & 183.6 \\
\hline 2 & R1 B0 H1 & 16.0 & 43.0 & 75.2 \\
\hline 4 & $\begin{array}{l}\text { R3 B2 H3, } \\
\text { diplophone }\end{array}$ & 9.0 & 18.0 & 149.6 \\
\hline 5 & $\mathrm{R} 2 \mathrm{~B} 1 \mathrm{H} 2$ & 12.0 & 30.0 & 144.4 \\
\hline
\end{tabular}

$R$, Roughness; $B$, breathiness; $H$, hoarseness. time. In an experimental rabbit model, Jacobs and colleagues ${ }^{13}$ showed that significant necrosis and cartilage resorption occurred, although grafts were reepithelialized within the first 3 weeks. The necrotic cartilage was largely replaced with new cartilage and fibrous tissue. Similar changes were reported by Oue and coworkers ${ }^{14}$ in autopsy studies of 18 infants who had previously received a costal cartilage tracheoplasty, with a mean period of 7.5 months after operation. The grafts were partly replaced by scar tissue and on their surface completely reepithelialized.

One could argue that the short-term need for tracheostomy might be a limitation of this technique; compared to a 3-month period of internal T-tube stenting, however, we consider this not relevant. In fact, the 3 patients who needed such a temporary utility tracheostomy were decannulated after 8, 9, and 14 days. Temporary swelling of the vocal cords is not an infrequent finding after operations of the glottic and subglottic airway. ${ }^{15}$ On the one hand, the surgical dissection can lead to a compromised venous and lymphatic drainage; on the other hand, direct mechanical irritation of the vocal cords can result in glottic swelling. A swollen glottis at the end of the operation can be handled in two ways. Some centers suggest placement of a nasotracheal tube and leaving the patient intubated for several days. This, however, has the potential disadvantage that the newly reconstructed airway can be damaged by compression and a tube by itself can perpetuate the swelling. Furthermore, the presence of the tube does not allow a postoperative monitoring of the degree of swelling. We therefore prefer the technique of a small temporary utility tracheostomy (minitracheostomy) distal to the reconstruction site. Small cannulas of size 5 or 6 are sufficient to maintain oxygenation. These patients are used to an increased airway resistance and therefore tolerate these small cannulas for a short period without problems. Once the swelling has resolved, the tracheostomy can easily be removed. An important technical aspect of placing a low tracheostomy is to use cannulas with a sufficient length. The distance between skin and trachea is usually too long to use cannulas with a standard length. In patient 5, the cannula was too short, which resulted in displacement during coughing. Soft tissue emphysema was the consequence; this resolved after placement of a longer cannula.

Another relevant technical aspect is the closure of the anterior laryngeal split. It is of utmost importance that the anterior commissure of the larynx is perfectly approximated and the vocal folds are readapted in a symmetric manner. In patient 4, a minimal displacement of the vocal folds $(<1 \mathrm{~mm})$ led to a mild impairment of her voice, although her vocal fold movements were grossly normal.

Although this series of patients is small, it owns the advantage of a full documentation of the resulting voice quality, something that is completely missing in most other 

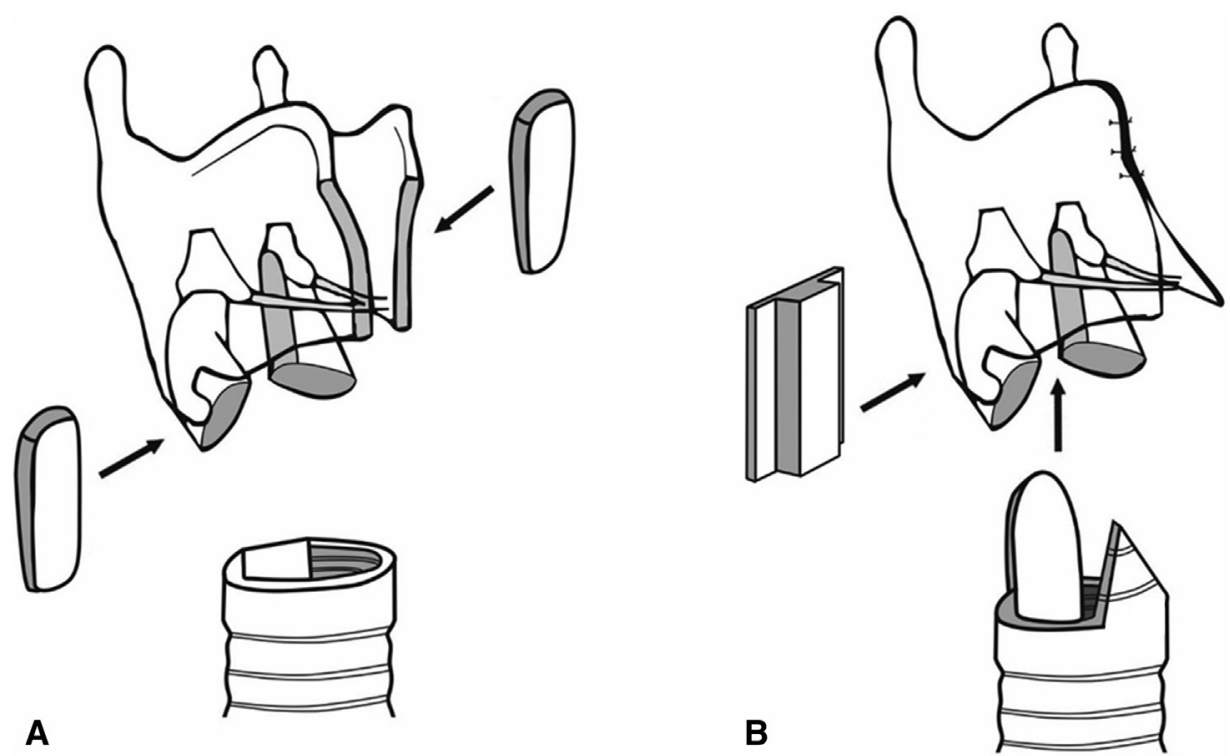

FIGURE 3. Diagram illustrates the main differences between the classical Couraud technique (A) and the modifications described in this case series (B).

case series. Only a mild impairment in the measured hoarseness parameters (roughness, breathiness) was found in subjective perceptive evaluation of voice quality. In addition to that, the fundamental frequency was significantly lowered after laryngotracheal reconstruction in all patients. This can be explained by the dissection of the cricothyroid muscles and changes in the configuration of the reconstructed larynges. A similar observation has been made in patients undergoing a classic cricotracheal resection. ${ }^{16,17}$

All our patients are treated by a dedicated team consisting of thoracic surgeons, phoniatricians, speech therapists, and anesthesiologists. Patients were evaluated preoperatively, underwent operation, and were followed up by members of the team. We believe that this interdisciplinary approach is pivotal to the management of complex airway disorders. A repair of the upper airways involving the glottis is a challenging procedure and requires a high surgical expertise. This is especially important because the reconstruction has to be successful at the first attempt. Reoperation after a failure of a laryngotracheal reconstruction is hardly possible, and a permanent tracheostomy is often the result of an improper repair.

This case series of 5 consecutive patients summarizes our initial experience with the modified technique of laryngotracheal reconstruction. It owns the clear advantage of omitting the need for postoperative stenting, which not only improves patients' quality of life but also avoids possible negative effects on the laryngeal mucosa (eg, granulation formation) that might occur from prolonged stenting. The functional results of this small series of patients was excellent not only in terms of improvement of the airway but also with regard to voice quality and swallowing.

\section{Webcast}

You can watch a Webcast of this AATS meeting presentation by going to: http://webcast.aats.org/2016/Video/Tuesday/ 05-17-16_Ballroom_IV_1420_Hoetzenecker-800.mp4.

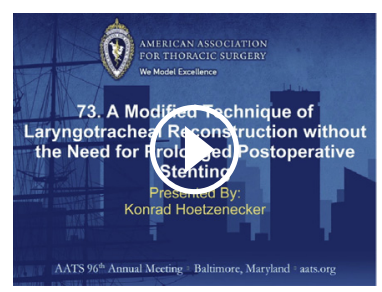

\section{Conflict of Interest Statement}

Authors have nothing to disclose with regard to commercial support.

\section{References}

1. Pearson FG, Cooper JD, Nelems JM, Van Nostrand AW. Primary tracheal anastomosis after resection of the cricoid cartilage with preservation of recurrent laryngeal nerves. J Thorac Cardiovasc Surg. 1975;70:806-16.

2. Grillo HC. Primary reconstruction of airway after resection of subglottic laryngeal and upper tracheal stenosis. Ann Thorac Surg. 1982;33:3-18.

3. Liberman M, Mathisen DJ. Tailored cricoplasty: an improved modification for reconstruction in subglottic tracheal stenosis. J Thorac Cardiovasc Surg. 2009; 137:573-8; discussion 578-9.

4. Morcillo A, Wins R, Gómez-Caro A, Paradela M, Molins L, Tarrazona V. Singlestaged laryngotracheal reconstruction for idiopathic tracheal stenosis. Ann Thorac Surg. 2013;95:433-4; discussion 439.

5. Couraud L, Hafez A, Velly JF, Gironnet I. Current reconstructive management of subglottic stenosis of the larynx with reference to sixty consecutively treated cases. Thorac Cardiovasc Surg. 1985;33:263-7.

6. Couraud L, Jougon JB, Velly JF. Surgical treatment of nontumoral stenoses of the upper airway. Ann Thorac Surg. 1995;60:250-9; discussion 259-60.

7. White DR, Cotton RT, Bean JA, Rutter MJ. Pediatric cricotracheal resection: surgical outcomes and risk factor analysis. Arch Otolaryngol Head Neck Surg. 2005; 131:896-9. 
8. Yamamoto K, Jaquet Y, Ikonomidis C, Monnier P. Partial cricotracheal resection for paediatric subglottic stenosis: update of the Lausanne experience with 129 cases. Eur J Cardiothorac Surg. 2015;47:876-82.

9. Monnier P. Pediatric airway surgery management of laryngotracheal stenosis in infants and children. Heidelberg: Springer; 2011. xvii.

10. Monnier P, Ikonomidis C, Jaquet Y, George M. Proposal of a new classification for optimising outcome assessment following partial cricotracheal resections in severe pediatric subglottic stenosis. Int J Pediatr Otorhinolaryngol. 2009;73:1217-21.

11. Grillo HC. Surgery of the trachea and bronchi. Lewiston (NY): BC Decker; 2004.

12. Hysi I, Kipnis E, Fayoux P, Copin MC, Zawadzki C, Jashari R, et al. Successful orthotopic transplantation of short tracheal segments without immunosuppressive therapy. Eur J Cardiothorac Surg. 2015;47:e54-61.

13. Jacobs IN, Podrebarac P, Boden SD, Chen M. Graft healing in laryngotracheal reconstruction: an experimental rabbit model. Ann Otol Rhinol Laryngol. 1999; 108:599-605.

14. Oue T, Kamata S, Usui N, Okuyama H, Nose K, Okada A. Histopathologic changes after tracheobronchial reconstruction with costal cartilage graft for congenital tracheal stenosis. J Pediatr Surg. 2001;36:329-33.

15. Wang H, Wright CD, Wain JC, Ott HC, Mathisen DJ. Idiopathic subglottic stenosis: factors affecting outcome after single-stage repair. Ann Thorac Surg. 2015;100:1804-11

16. Houlton JJ, de Alarcon A, Johnson K, Meinzen-Derr J, Brehm SB, Weinrich B, et al. Voice outcomes following adult cricotracheal resection. Laryngoscope. 2011;121:1910-4.

17. Smith ME, Roy N, Stoddard K, Barton M. How does cricotracheal resection affect the female voice? Ann Otol Rhinol Laryngol. 2008;117:85-9.

Key Words: airway surgery, stenosis, trachea, subglottic, laryngotracheal

\section{Discussion}

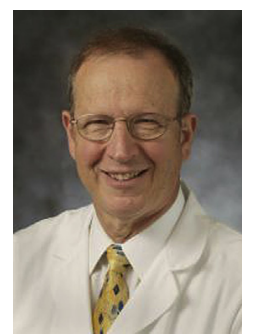

Dr Joel D. Cooper (Philadelphia, Pa). Thank you. I appreciate the opportunity to discuss the paper and congratulate $\mathrm{Dr}$ Hoetzenecker and his colleagues on an excellent contribution.

I was originally sent the manuscript to review before it was submitted to the Association for presentation; subsequently, when it was submitted and accepted, I was invited to discuss the paper. I want to make some comments about the paper and some observations and ask a few questions, and I have 2 minutes in which to do it, so I thought I would start off by reading my original review of the manuscript, which in my case ordinarily consists of 3 to 4 pages of critical comments.

"It was an absolute delight to review the manuscript by Hoetzenecker and colleagues. The manuscript is an important contribution, highly relevant to a small but distinct and important group of individuals with benign laryngotracheal stenosis involving both the glottic and the subglottic region. The article was well referenced, very clearly and precisely presented, with good illustrations, and the best, most objective assessment of outcome function I have ever seen for this type of problem. In more than 40 years of reviewing manuscripts, I cannot recall a paper that I consider more perfectly written than this one, to the point that I am unable to offer even one constructive criticism. I would appreciate if you would convey my congratulations to the authors for their excellent contribution, which I recognize is of importance only to a relatively small number of experts in the field of laryngotracheal reconstruction." That was my review.

Now, to be perfectly honest, my review did trouble me. I will have to leave it to the audience, but I think I have a bit of a reputation as being a confirmed critic, both of my own work and of others, and I will have to leave it to you to decide whether the paper is indeed as good as I said or whether, unfortunately, senescence is gradually creeping into my life. I do know that I have reached the stage where, as my dad used to say, "My future is behind me," and this makes me more interested in history. One of the things I appreciate is the attribution that the authors gave to the previous surgeons who contributed to the development of this procedure. It is a good story.

Originally in the 1970s, Gerwatt and Bryce at the University of Toronto described laryngotracheal resection with primary anastomosis, but only in people with bilateral recurrent nerve damage, and therefore the procedure had limited applicability. In 1975, Dr Pearson and some of the rest of us in Toronto presented the technique of a laryngotracheal resection, reaming out the cricoid plate but leaving the posterior perichondrium to avoid injury to the recurrent nerves and allowing resection of subglottic strictures with preservation of the nerves. A few years later, Dr Grillo adopted that procedure and included a posterior mucosal flap fashioned from the membranous wall of the trachea. Subsequently, Louis Couraud in Marseille extended the resection to the point where he could perform the anastomosis at the level of the vocal cords and stent it. I think he used to leave a nasotracheal tube in for weeks, if not months. Then Phillipe Monnier in Lausanne adapted it to pediatrics and has written some excellent work on its application. He developed, as noted by the authors, the insertion of a posterior cartilage graft to stabilize the posterior vertical split of the cricoid plate, but this procedure required postoperative stenting of the anastomosis. Now the technique presented here provides for stabilizing the cartilage graft and covering it with a Grillo-type mucosal flap, avoiding the need for postoperative stenting. I think it's a very interesting and important contribution, and one that once again demonstrates the value of multidisciplinary cooperation. Dr Pearson worked closely with Dr Bryce, the head of ear, nose, and throat (ENT) surgery at the University of Toronto, and Dr Grillo similarly worked closely with Dr Bill Montgomery of the Massachusetts Eye and Ear Infirmary. I noticed that Dr Klepetko, who is the senior author of this paper, has really carried this collaboration to a much higher level, because, at least on the manuscript I received, he was listed not as a member of the thoracic surgery department but as a member of the ENT department.

I do have a question or two for Dr Hoetzenecker. First, is it necessary to do the full anterior laryngofissure? I sometimes do it up to the point of the vocal process, which avoids the possibility of a displaced vocal commissure, which you refer to in your article. So do you always have to do the full anterior laryngofissure? 
Second, and perhaps most importantly, what do you advise others not to do? The subglottic strictures that we see so often have been complicated by lasering, dilatations, and other things that interventional pulmonologists and other ENT people may have done. What do you recommend not be done to make it easier for you to do a repair of this very complicated situation?

Finally, it might be very helpful in these days of wonderful science if you could take some rib cartilages from a cadaver, carve them the way you like, put them in a 3-dimensional printer, and be able to send some of us a little plastic model of exactly what you do, because, to be perfectly honest, I might see one of these situations only every 2 years, even though we do a lot of laryngotracheal resections.

Finally, a T-tube is actually a very good stent. I think that your primary contribution is not just the avoidance of the stenting, which in young people, even if it is transglottic, is actually quite well tolerated. I think the contribution is that you have added a very nice enhancement and a better way of doing this type of resection in a single stage.

I congratulate you and thank the Association for the opportunity of discussing the paper.

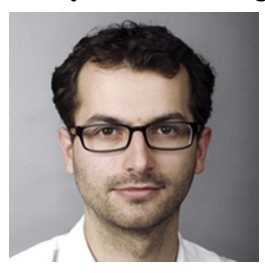

Dr Hoetzenecker. Thank you for your very kind words, Dr Cooper.

With respect to your first question if a full anterior split is necessary, I think this really depends on the individual anatomy of the glottis and on the problem we are trying to resect. I agree that for maybe half of the cases, a partial anterior split ending at the level of the insertion of the vocal cords is sufficient to get a good view on the posterior scarring. In some cases, however, and this is especially true for pediatric cases, you need a perfect exposure posteriorly, and for those cases a complete split is necessary. We usually carefully review the computed tomographic scan preoperatively. During the operation we look from below and then decide whether we have to do a full split or just a partial split. When we do a full split, the upper third of the split has to be closed again at the end of the operation so that the vocal cords come together, otherwise the patient will be aphonic after the operation. This is a very important aspect.

With respect to your second question, I think that we as surgeons would always like to operate on patients who have not been pretreated with endoscopic techniques, such as lasering or dilatation. Those pretreatments usually result in more difficult repairs and the need for more extended techniques, and to be honest everything gets more and more complicated. What we do in Vienna is review all of those cases together with our ENT colleagues, and for those kind of stenoses that are high up, we go for primary surgery. It is interesting that you actually mentioned a 3-dimensional printer. I had a discussion yesterday with Dr Yasufuku from Toronto. He has access to a 3-dimensional printer, and we talked about a similar approach to the one you just proposed. I am very much looking forward to this project.

Dr Cooper. Thanks. If I could just add one thing, I failed to mention anesthesia. You emphasized that. The team is ENT, thoracic surgery, and anesthesia, 3 critical elements, and I'm sure you feel the same way.

Dr Hoetzenecker. I perfectly agree, yes. 